\title{
Doxorubicin-induced cardiomyopathy: TERT gets to the heart of the matter
}

\author{
Bart De Geest ${ }^{1}$, Mudit Mishra ${ }^{2}$
}

${ }^{1}$ Centre for Molecular and Vascular Biology, Catholic University of Leuven, Leuven, Belgium ${ }^{2}$ Department of Cardiothoracic Surgery, University Medical Center Utrecht, Utrecht, the Netherlands

\section{Correspondence:}

Bart De Geest MD, PhD

Centre for Molecular and Vascular Biology

Department of Cardiovascular Sciences

Catholic University of Leuven

Campus Gasthuisberg

Herestraat 49 bus 911

3000 Leuven

Belgium

Tel.: 003216372059

Fax.: 003216345990

E-mail: bart.degeest@kuleuven.be 
The chemotherapeutic doxorubicin is the most frequently prescribed anticancer drug and is given as a single agent or in combination with other antitumor drugs. Administration of doxorubicin can lead to both short- and long-term cardiotoxic effects, ranging from subclinical alterations of myocardial structure and function to severe cardiomyopathy and heart failure that may result in heart transplantation or death. In this issue of Molecular Therapy, Chatterjee et al. ${ }^{1}$ address this key challenge in cardio-oncology by demonstrating that AAV9mediated gene transfer of murine telomerase reverse transcriptase (Tert) under control of the cardiomyocyte-specific cardiac troponin T promoter (AAV9-Tert) prevents cardiac atrophy and systolic dysfunction in a murine model of chronic doxorubicin cardiotoxicity.

Doxorubicin is a potent cytotoxic antibiotic originally isolated from Streptomyces peucetius caesius and belongs to the anthracycline group of chemotherapeutic agents. This drug is used in the treatment of a wide variety of both pediatric and adult malignancies, including medulloblastoma, leukemia, several carcinomas (breast cancer, stomach cancer, lung cancer, bladder cancer, kidney cancer, ovarian cancer, thyroid cancer), sarcoma, lymphoma and multiple myeloma. The most important risk factor for anthracycline-induced cardiotoxicity is the cumulative dose and the incidence of doxorubicin-induced cardiomyopathy in patients who received a cumulative dose exceeding $600 \mathrm{mg} / \mathrm{m}^{2}$ is above $30 \%{ }^{2}$

Doxorubicin accumulates predominantly in mitochondria and nuclei, ${ }^{3}$ and mitochondrial toxicity is a key factor in its cardiotoxicity. ${ }^{2}$ Doxorubicin redox cycles primarily on complex I (NADH:ubiquinone oxidoreductase) of the mitochondrial respiratory chain. ${ }^{2}$ One-electron reduction of the quinone moiety present in doxorubicin results in the formation of a free radical semiquinone that regenerates the parent quinone by reducing oxygen to superoxide radical (Figure 1). This initiates a reaction cascade leading to the formation of very reactive oxygen species, oxidative damage, and activation of mitochondrial-dependent apoptotic pathways. Given that mitochondria are highly abundant in cardiomyocytes, this may explain the cardio-selective toxicity of doxorubicin. Whereas the presence of doxorubicin in the mitochondria causes direct toxicity, at least some of the mitochondrial toxicity is indirect and secondary to effects of doxorubicin on the cell nucleus. ${ }^{2}$ The complex of doxorubicin and topoisomerase $\| \beta$ in the nucleus can lead to inhibition of mitochondrial biogenesis and gene expression resulting in secondary inhibition of oxidative phosphorylation. ${ }^{2}$ Moreover, 
signaling occurs between the nucleus and mitochondria and consists of a complex network of events involving proteins and RNAs. This network includes TERT.

Telomerase is a ribonucleoprotein containing two major components: TERT and an RNA subunit telomerase RNA component (TERC). The proper telomerase holoenzyme assembly requires the enzymatic contribution of a number of accessory proteins with most of them acting on the RNA component of the enzyme. TERT is completely silenced in adult cardiomyocytes, as is the case in most differentiated human cells, by a mechanism of transcriptional repression. The canonical function of telomerase is to add telomere repeat sequences to the 3 '-end of telomeres, thereby ensuring genomic stability and protecting cells against induction of replicative senescence or apoptosis.

At the microscopic level, Chatterjee et al. ${ }^{1}$ show that AAV9-Tert gene transfer completely blocked the doxorubicin-induced cardiomyocyte atrophy and apoptosis in the myocardium, rescued severe misalignment of the sarcomeres upon doxorubicin treatment, and counteracted increased mitochondrial fission induced by doxorubicin. ${ }^{1}$ Furthermore, the authors generated human cardiomyocytes from induced pluripotent stem cells (hiPSC-CMs) based on a differentiation protocol that included a selection step as well as a 60-day maturation period. Neither human TERT mRNA expression nor detectable telomerase activity was present in these differentiated hiPSC-CMs. Following AAV6-TERT gene transfer into these hiPSC-CMs, TERT protein was observed in the mitochondrial compartment and this translocation increased under acute doxorubicin stress for 48 hours. AAV6-TERT gene transfer counteracted doxorubicin-induced apoptosis, maintained the mitochondrial content upon doxorubicin treatment, reduced reactive oxygen species levels, and even restored mitochondrial function under conditions of doxorubicin stress.

The effect of AAV9-Tert gene transfer on chronic doxorubicin cardiotoxicity in vivo and of AAV6-TERT gene transfer on acute doxorubicin toxicity in hiPSC-CMs in vitro was independent of the canonical function of telomerase since telomere length was unaltered. ${ }^{1}$ TERT has multiple non-canonical roles that are independent of its telomere elongation activity and include signal transduction, gene expression regulation, and protection against oxidative damage. Under stress conditions, TERT is exported out of the nucleus and imported into 
mitochondria, where it may exert protective effects. TERT nuclear export is regulated by phosphorylation. ${ }^{4}$ A mitochondrial localization sequence at the $\mathrm{N}$ terminus of TERT has been identified. In the mitochondrial matrix, overexpressed TERT has been demonstrated to bind to the mitochondrial DNA at the coding sequences for NADH:ubiquinone oxidoreductase (complex I) subunit 1 and 2 (ND1 and ND2). ${ }^{5}$ Haendeler et al. ${ }^{5}$ have previously shown that mitochondrial TERT increases respiratory chain activity and protects against oxidative stressinduced damage. ${ }^{5}$ Effects of TERT overexpression may be dependent on degree of overexpression, cell type, and species. Chatterjee et al. ${ }^{1}$ clearly demonstrate mitochondrial import of TERT and the mitochondrial effects of TERT in hiPSC-CMs in vitro. Likewise, it is plausible that these mitochondrial protective mechanisms are also operational in cardiomyocytes in their murine model in vivo. In contrast to TERT, human TERC is expressed in most normal human cells. TERC has several functions other than serving as template for telomere extension and can also be imported into the mitochondria. Whether this translocation process of TERC has any role in cardiac biology remains to be established.

The interval between the last administration of doxorubicin and quantification of functional and structural endpoints in mice was one week in the study of Chatterjee et al., ${ }^{1}$ which is the minimum to be considered representative of chronic cardiotoxicity. Chronic progressive cardiotoxicity in humans, which presents as a dilated cardiomyopathy in adults and as a restrictive or dilated cardiomyopathy in children, becomes apparent within one year of the completion of treatment (early-onset chronic cardiotoxicity) or several years after completion of chemotherapy (late-onset chronic cardiotoxicity). Doxorubicin and metabolites may persist in cardiac tissue for a period that is significantly longer than in plasma but persistent alterations in mitochondrial function cannot be related to the physical presence of the drug. In addition, the estimated half-life for the turnover of cardiac mitochondria proteins is 16 to 18 days. ${ }^{2}$ Several mechanisms for cardiac doxorubicin toxicity memory have been proposed. ${ }^{2}$ A first mechanism is the oxidation of mitochondrial DNA and subsequent reduction in mitochondrial DNA copy number. Second, alteration of the nuclear epigenetic landscape may occur indirectly related to disturbed mitochondrial metabolism, which alters the availability of methyl, acetyl, or phosphate donors for epigenetic regulation. Third, persistent effects of cardiomyocyte apoptosis and reduced cardiac mass induced by doxorubicin may have persistent effects in particular in children. Therefore, the question is whether cardiomyocyte 
TERT expression induced by gene transfer or by transient reactivation of the TERT promoter at the time of doxorubicin administration can abrogate cardiac doxorubicin toxicity memory.

This study of Chatterjee et al. ${ }^{1}$ is not only a very important contribution to the field of experimental cardio-oncology by its salient observations at the functional and structural level in vivo, but it also represents a remarkable application of a non-canonical function of TERT. TERT gets to the heart of the matter by its physical localization in the mitochondria and its effects on oxidative stress, mitochondrial function, and potentially on cardiac doxorubicin toxicity memory.

\section{REFERENCES}

1. Chatterjee, S., Hofer, T., Costa, A., Lu, D., Batkai, S., Gupta, S.K., et al. (2021). Telomerase therapy attenuates cardiotoxic effects of doxorubicin. Mol. Ther 29,

2. Wallace, K.B., Sardão, V.A., and Oliveira, P.J. (2020). Mitochondrial Determinants of Doxorubicin- Induced Cardiomyopathy. Circ. Res. 126, 926-941.

3. Cova, D., De Angelis, L., Monti, E., and Piccinini, F. (1992). Subcellular distribution of two spin trapping agents in rat heart: possible explanation for their different protective effects against doxorubicininduced cardiotoxicity. Free Radic. Res. Commun. 15, 353-360.

4. Zheng, Q., Huang, J., and Wang, G. (2019). Mitochondria, Telomeres and Telomerase Subunits. Front. Cell Dev. Biol. 7, 274.

5. Haendeler, J., Dröse, S., Büchner, N., Jakob, S., Altschmied, J., Goy, C., Spyridopoulos, I., Zeiher, A.M., Brandt, U., and Dimmeler, S. (2009). Mitochondrial telomerase reverse transcriptase binds to and protects mitochondrial DNA and function from damage. Arterioscler. Thromb. Vasc. Biol. 29, 929-935. 


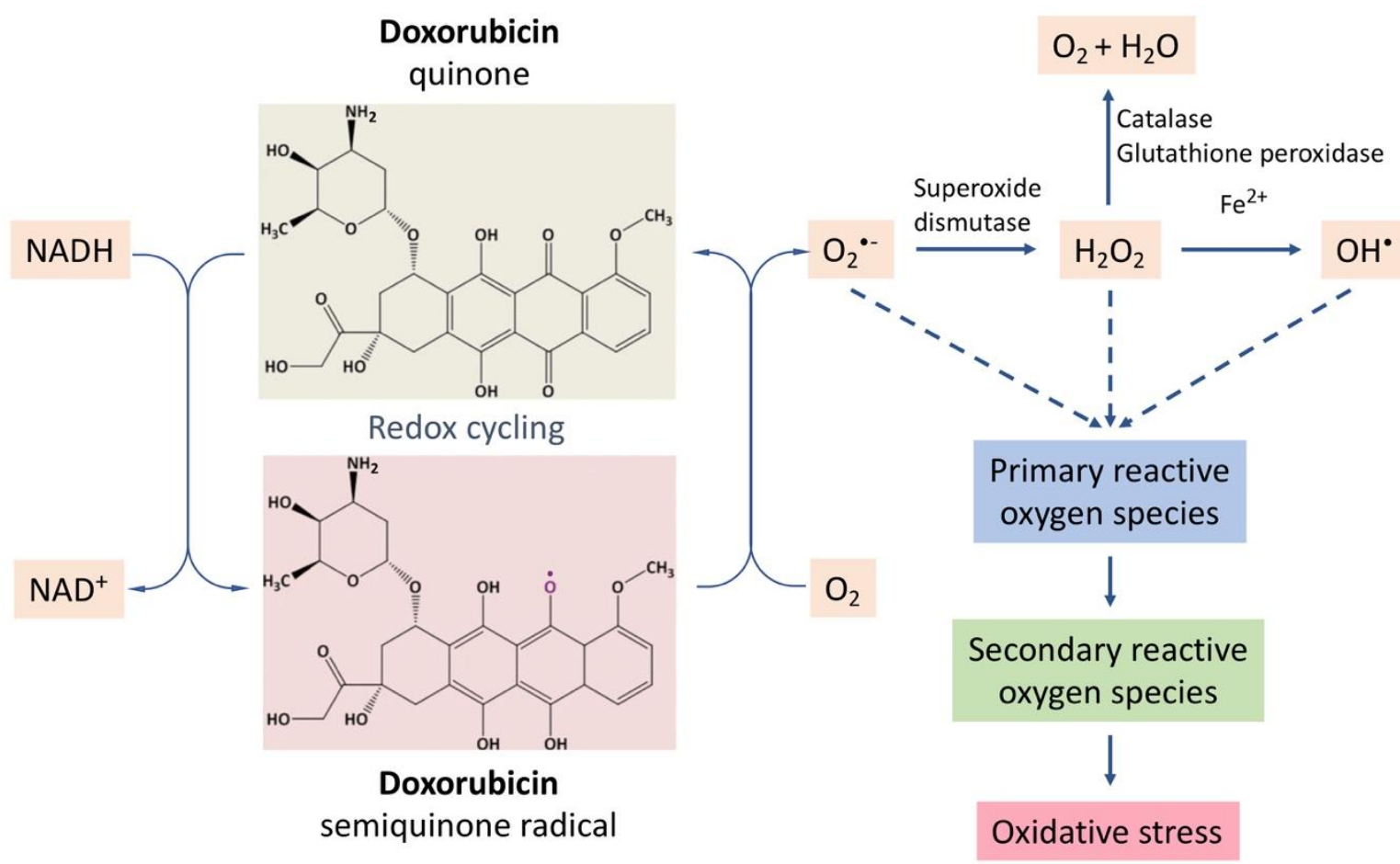

Figure 1. Redox cycling of doxorubicin in the mitochondria. Doxorubicin is bioactivated by complex I of the mitochondrial respiratory chain at the expense of NADH. This is followed by the rapid re-oxidation of the semiquinone radical in the presence of molecular oxygen to generate superoxide anion-free radicals. Subsequently, further primary reactive oxygen species and secondary reactive oxygen species are produced leading to oxidative stress. 